\title{
Article
}

\section{On the existence of positive solutions of a state-dependent neutral functional differential equation with two state-delay functions}

\author{
El-Sayed, A. M. A ${ }^{1}$, Hamdallah, E. M. $\mathbf{A}^{\mathbf{1}}$ and Ebead, H. R ${ }^{1, *}$ \\ 1 Faculty of Science, Alexandria University, Alexandria, 21500, Egypt. \\ * Correspondence: hanaarezqalla@alexu.edu.eg
}

Received: 2 September 2020; Accepted: 21 October 2020; Published: 14 December 2020.

\begin{abstract}
In this paper, we study the existence of positive solutions for an initial value problem of a state-dependent neutral functional differential equation with two state-delay functions. The continuous dependence of the unique solution will be proved. Some especial cases and examples will be given.
\end{abstract}

Keywords: Neutral differential equations, state-dependent, existence of positive solutions, continuous dependence.

MSC: 35G16, 74Dxx, 35B40.

\section{Introduction}

$\mathbf{T}$ he differential and integral equations with deviating arguments usually involve the deviation of the argument only the time itself, however, another case, in which the deviating arguments depend on both the state variable $x$ and the time $t$ is important in theory and practice. This kind of equations is called self-reference or state dependent equations. Differential equations with state-dependent delays attract interests of specialists since they widely arise from application models, such as two-body problem of classical electrodynamics also have may applications in the class of problems that have past memories, for example in hereditary phenomena see [1-4]. For papers studying such kind of equations, see for example [5-16] and references therein.

One of the first papers studying this class of equations was introduced by Buicá [7], the author proved the existence and the uniqueness of the solution of the initial value problem

$$
\begin{aligned}
\frac{d x(t)}{d t} & =f(t, x(x(t))), \quad t \in[a, b], \\
x(0) & =x_{0},
\end{aligned}
$$

where $f \in C([a, b] \times[a, b])$ and satisfied Lipshitz condition.

EL-Sayed and Ebead [13] relaxed the assumptions of Buicá and generalized their results, they studied the functional integral equation of the more general form

$$
x(t)=f\left(t, \int_{0}^{t} g(s, x(x(s))) d s\right), \quad t \in[0, T],
$$

where $g$ satisfies Carathéodory condition.

El-Sayed and Ahmed [9] studied the existence of solutions and its continuous dependence of the initial value problem

$$
\begin{aligned}
\frac{d}{d t} x(t) & =f\left(t, x\left(\int_{0}^{\phi(t)} g(s, x(s)) d s\right)\right), \text { a.e. } t \in(0, T] \\
x(0) & =x_{0}
\end{aligned}
$$


where $g:[0, T] \times R^{+} \rightarrow R^{+}$is continuous and $g(t, x(t)) \leq 1$ and $\phi(t) \leq t$.

El-Sayed and Ebead $[11,12]$ studied the existence of solution and its continuous dependence of the initial value problem of the delay-refereed differential equation

$$
\begin{aligned}
\frac{d}{d t} x(t) & =f(t, x(g(t, x(t)))), \text { a.e. } t \in(0, T] \\
x(0) & =x_{0},
\end{aligned}
$$

with the two cases of state-delay functions

(i) $g:[0, T] \times R^{+} \rightarrow[0, T]$ is continuous and $g(t, x(t)) \leq t$,

(ii) $g:[0, T] \times[0, T] \rightarrow[0, T]$ is continuous and $g(t, x(\phi(t))) \leq x(\phi(t))$.

Here we shall study the initial value problem of state-dependent neutral functional differential equation with two state-delay functions

$$
\frac{d}{d t}\left[x(t)-f_{1}\left(t, x\left(g_{1}(t,(x(\phi(t))))\right)\right)\right]=f_{2}\left(t, x\left(g_{2}(t, x(\phi(t)))\right)\right), \text { a.e. } t \in[0, T],
$$

with the initial data

$$
x(0)=f_{1}\left(0, x\left(g_{1}(0, x(0))\right)\right),
$$

where $g_{i}, i=1,2$ are continuous and $g_{1}(t, x) \leq \phi(t)$ and $g_{1}(t, x) \leq x(t)$.

Our aim in this work is to study the existence of at least one and exactly one positive solution of the Problem (1)-(2). The continuous dependence of the unique solution on the two functions $g_{1}$ and $g_{2}$ will be proved. To illustrate our results some examples will be given.

In order to achieve our goal, we study the existence of positive solutions $x \in C[0 ; T]$ for the state-dependent functional integral equation

$$
x(t)=f_{1}\left(t, x\left(g_{1}(t,(x(\phi(t))))\right)\right)+\int_{0}^{t} f_{2}\left(s, x\left(g_{2}(s, x(\phi(s)))\right)\right) d s \quad t \in[0, T],
$$

and we will show later that this integral equation is equivalent to the initial value Problem (1)-(2).

\section{Existence of solutions}

Here we study the existence of solutions $x \in C[0, T]$ for the integral Equation (3) under the following assumptions

(1) $f_{1}:[0, T] \times[0, T] \rightarrow R^{+}$is continuous and there exist two positive constants $k_{1}$ and $k_{2}$ such that

$$
\left|f_{1}\left(t_{2}, x\right)-f_{1}\left(t_{1}, y\right)\right| \leq k_{1}\left|t_{2}-t_{1}\right|+k_{2}|x-y| .
$$

(2) $g_{1}:[0, T] \times[0, T] \rightarrow[0, T]$ is continuous and there exist the two positive constants $k_{3}, k_{4}$ such that

$$
\left|g_{1}\left(t_{2}, x\right)-g_{1}\left(t_{1}, y\right)\right| \leq k_{3}\left|t_{2}-t_{1}\right|+k_{4}|x-y|
$$

and $g_{1}(t, x) \leq \phi(t)$

(3) $f_{2}:[0, T] \times[0, T] \rightarrow R^{+}$satisfies Carathéodory condition i.e. $f(t, x)$ is measurable in $t$ for all $x \in C[0, T]$ and continuous in $x$ for almost all $t \in[0, T]$.

(4) There exists a bounded measurable function $m:[0, T] \rightarrow R^{+}, m(t) \leq A$, and a constant $k_{5} \geq 0$ such that

$$
f_{2}(t, x) \leq m(t)+k_{5} x
$$

(5) $g_{2}:[0, T] \times[0, T] \rightarrow[0, T]$ is continuous and $g_{2}(t, x) \leq x$

(6) $\phi:[0, T] \rightarrow[0, T], \phi(0)=0$ and

$$
|\phi(t)-\phi(s)| \leq|t-s|,
$$

which implies that $\phi(t) \leq t$. 
(7) There exists a real positive solution $L \in(0,1)$ of the equation

$$
k_{2} k_{4} L^{2}+\left(k_{2} k_{3}-1\right) L+M+k_{1}=0,
$$

where $M=A+k_{5} T$.

(8) $L T+x(0) \leq T$.

Now we are in a position to prove the following existence theorem.

Theorem 1. Let the assumptions (1) - (8) be satisfied, then the state-dependent integral Equation (3) has at least one positive solution $x \in C[0, T]$.

Proof. Let $X=C[0, T]$ be the class of real valued continuous functions defined on $[0, T]$. Define the subset $S_{L}$ of $X$ by

$$
S_{L}=\left\{x \in X:\left|x\left(t_{2}\right)-x\left(t_{1}\right)\right| \leq L\left|t_{2}-t_{1}\right|\right\} .
$$

It is clear that $S_{L}$ is nonempty, closed, bounded and convex subset of $C[0, T]$.

Now define the operator $F$ associated with Equation (3) by

$$
F x(t)=f_{1}\left(t, x\left(g_{1}(t, x(\phi(t)))\right)\right)+\int_{0}^{t} f_{2}\left(s, x\left(g_{2}(s, x(\phi(s)))\right)\right) d s, t \in[0, T] .
$$

It is clear that $\mathrm{F}$ makes sense and well-defined.

Now, first we prove that the class of functions $\{F x\}$ is uniformly bounded on the set $S_{L}$. Let $x \in X$, then for $t \in[0, T]$, we obtain

$$
\begin{aligned}
|F x(t)| & =\left|f_{1}\left(t, x\left(g_{1}(t, x(\phi(t)))\right)\right)+\int_{0}^{t} f_{2}\left(s, x\left(g_{2}(s, x(\phi(s)))\right)\right) d s\right| \\
& \leq\left|f_{1}\left(t, x\left(g_{1}(t, x(\phi(t)))\right)\right)\right|+\int_{0}^{t}\left|f_{2}\left(s, x\left(g_{2}(s, x(\phi(s)))\right)\right)\right| d s .
\end{aligned}
$$

Using assumptions (1),(2) and (6) we can get

$$
\begin{aligned}
\left|f_{1}\left(t, x\left(g_{1}(t, x(\phi(t)))\right)\right)\right| & =\left|f_{1}\left(t, x\left(g_{1}(t, x(\phi(t)))\right)\right)-f_{1}\left(0, x\left(g_{1}(0, x(0))\right)\right)\right|+\left|f_{1}\left(0, x\left(g_{1}(0, x(0))\right)\right)\right| \\
& \leq k_{1} T+k_{2}\left|x\left(g_{1}(t, x(\phi(t)))\right)-x\left(g_{1}(0, x(0))\right)\right|+\left|f_{1}\left(0, x\left(g_{1}(0, x(0))\right)\right)\right| \\
& \leq k_{1} T+k_{2} L\left|g_{1}(t, x(\phi(t)))-g_{1}(0, x(0))\right|+x(0) \\
& \leq k_{1} T+k_{2} L\left(k_{3} T+k_{4}|x(\phi(t))-x(0)|\right)+x(0) \\
& \leq k_{1} T+k_{2} L\left(k_{3} T+k_{4} L \phi(t)\right)+x(0) \\
& \leq\left(k_{1}+k_{2} k_{3} L+k_{2} k_{4} L^{2}\right) T+x(0) .
\end{aligned}
$$

Using assumptions (3) and (4) we can get

$$
\begin{aligned}
\left|f_{2}\left(t, x\left(g_{2}(s, x(\phi(t)))\right)\right)\right| & \leq k_{5} x\left(g_{2}(s, x(\phi(t)))\right)+m(t) \\
& \leq k_{5}\left\{\left|x\left(g_{2}(s, x(\phi(t)))\right)-x(0)\right|+x(0)\right\}+m(t) \\
& \leq k_{5}\left(L g_{2}(s, x(\phi(t)))+x(0)\right)+A \\
& \leq k_{5}(L x(\phi(t))+x(0))+A .
\end{aligned}
$$

Now from (4) and (5) and by assumption (8), we get

$$
\begin{aligned}
|F x(t)| & \leq\left(k_{1}+k_{2} k_{3} L+k_{2} k_{4} L^{2}\right) T+x(0)+\int_{0}^{t}\left(k_{5}(L x(\phi(s))+x(0))+A\right) d s \\
& \leq\left(k_{1}+k_{2} k_{3} L+k_{2} k_{4} L^{2}\right) T+x(0)+\int_{0}^{t}\left(k_{5}(L T+x(0))+A\right) d s \\
& \leq\left(k_{1}+k_{2} k_{3} L+k_{2} k_{4} L^{2}\right) T+x(0)+\left(k_{5} T+A\right) \int_{0}^{t} d s
\end{aligned}
$$




$$
\begin{aligned}
& \leq\left(k_{1}+k_{2} k_{3} L+k_{2} k_{4} L^{2}+M\right) T+x(0) \\
& =L T+x(0) \leq T .
\end{aligned}
$$

This proves that the class of functions $\{F x\}$ is uniformly bounded on the set $S_{L}$.

Next, we prove that $F: S_{L} \rightarrow S_{L}$ and the class of functions $\{F x\}$ is equi-continuous on the set $S_{L}$. Let $x \in S_{L}$ and $t_{1}, t_{2} \in[0, T]$ with $t_{1}<t_{2}$ such that $\left|t_{2},-t_{1}\right|<\delta$, then

$$
\begin{aligned}
\left|F x\left(t_{2}\right)-F x\left(t_{1}\right)\right|= & \mid f_{1}\left(t_{2}, x\left(g_{1}\left(t_{2}, x\left(\phi\left(t_{2}\right)\right)\right)\right)\right)+\int_{0}^{t_{2}} f_{2}\left(s, x\left(g_{2}(s, x(\phi(s)))\right)\right) d s \\
& -f_{1}\left(t_{1}, x\left(g_{1}\left(t_{1}, x\left(\phi\left(t_{1}\right)\right)\right)\right)\right)-\int_{0}^{t_{1}} f_{2}\left(s, x\left(g_{2}(s, x(\phi(s)))\right)\right) d s \mid \\
\leq & \left|f_{1}\left(t_{2}, x\left(g_{1}\left(t_{2}, x\left(\phi\left(t_{2}\right)\right)\right)\right)\right)-f_{1}\left(t_{1}, x\left(g_{1}\left(t_{1}, x\left(\phi\left(t_{1}\right)\right)\right)\right)\right)\right|+\int_{t_{1}}^{t_{2}}\left|f_{2}\left(s, x\left(g_{2}(s, x(\phi(s)))\right)\right)\right| d s \\
\leq & k_{1}\left|t_{2}-t_{1}\right|+k_{2}\left|x\left(g_{1}\left(t_{2}, x\left(\phi\left(t_{2}\right)\right)\right)\right)-x\left(g_{1}\left(t_{1}, x\left(\phi\left(t_{1}\right)\right)\right)\right)\right|+\int_{t_{1}}^{t_{2}}\left(k_{5} T+A\right) d s \\
\leq & k_{1}\left|t_{2}-t_{1}\right|+k_{2}\left|x\left(g_{1}\left(t_{2}, x\left(\phi\left(t_{2}\right)\right)\right)\right)-x\left(g_{1}\left(t_{1}, x\left(\phi\left(t_{1}\right)\right)\right)\right)\right|+M\left|t_{2}-t_{1}\right| .
\end{aligned}
$$

Using assumptions (2) and $x \in S_{L}$, we can get

$$
\begin{aligned}
\left|x\left(g_{1}\left(t_{2}, x\left(\phi\left(t_{2}\right)\right)\right)\right)-x\left(g_{1}\left(t_{1}, x\left(\phi\left(t_{1}\right)\right)\right)\right)\right| & \left.\left.\leq L \mid g_{1}\left(t_{2}, x\left(\phi\left(t_{2}\right)\right)\right)\right)-g_{1}\left(t_{1}, x\left(\phi\left(t_{1}\right)\right)\right)\right) \mid \\
& \left.\left.\leq L k_{3}\left|t_{2}-t_{1}\right|+L k_{4} \mid x\left(\phi\left(t_{2}\right)\right)\right)-x\left(\phi\left(t_{1}\right)\right)\right) \mid \\
& \leq L k_{3}\left|t_{2}-t_{1}\right|+L^{2} k_{4}\left|\phi\left(t_{2}\right)-\phi\left(t_{1}\right)\right| \\
& \leq L k_{3}\left|t_{2}-t_{1}\right|+L^{2} k_{4}\left|t_{2}-t_{1}\right| .
\end{aligned}
$$

Then, we have

$$
\begin{aligned}
\left|F x\left(t_{2}\right)-F x\left(t_{1}\right)\right| & \leq k_{1}\left|t_{2}-t_{1}\right|+k_{2}\left(L k_{3}\left|t_{2}-t_{1}\right|+L^{2} k_{4}\left|t_{2}-t_{1}\right|\right)+M\left|t_{2}-t_{1}\right| \\
& =\left(k_{2} k_{4} L^{2}+k_{2} k_{3} L+k_{1}+M\right)\left|t_{2}-t_{1}\right| \\
& =L\left|t_{2}-t_{1}\right| .
\end{aligned}
$$

Hence, we proved that $F: S_{L} \rightarrow S_{L}$ and the class of functions $\{F x\}$ is equi-continuous on the set $S_{L}$. Applying Arzela-Ascoli Theorem [17], we deduce that $F$ is compact operator. Now we show that $F$ is continuous. Let $\left\{x_{n}\right\} \subset S_{L}, x_{n} \rightarrow x$ on $[0, T]$, i.e. $\left|x_{n}(\phi(t))-x(\phi(t))\right| \leq \epsilon_{1}$ this implies that $\left|x_{n}\left(g_{i}(t, x(\phi(t)))\right)-x\left(g_{i}(t, x(\phi(t)))\right)\right| \leq \epsilon_{2}$ for arbitrary $\epsilon_{1}, \epsilon_{2} \geq 0, i=1,2$, then

$$
\begin{aligned}
& \left|x_{n}\left(g_{i}\left(t, x_{n}(\phi(t))\right)\right)-x\left(g_{i}(t, x(\phi(t)))\right)\right| \\
& \leq\left|x_{n}\left(g_{i}\left(t, x_{n}(\phi(t))\right)\right)-x_{n}\left(g_{i}(t, x(\phi(t)))\right)\right|+\left|x_{n}\left(g_{i}(t, x(\phi(t)))\right)-x\left(g_{i}(t, x(\phi(t)))\right)\right| \\
& \leq L\left|g_{i}\left(t, x_{n}(\phi(t))\right)-g_{i}(t, x(\phi(t)))\right|+\left|x_{n}\left(g_{i}(t, x(\phi(t)))\right)-x\left(g_{i}(t, x(\phi(t)))\right)\right| \\
& \leq \epsilon, \quad i=1,2 .
\end{aligned}
$$

Then

$$
x_{n}\left(g_{i}\left(t, x_{n}(\phi(t))\right)\right) \rightarrow x\left(g_{i}(t, x(\phi(t)))\right) \text { in } S_{L}, \quad i=1,2
$$

and by using the continuity of the functions $f_{1}$, we obtain

$$
f_{1}\left(t, x_{n}\left(g_{1}\left(t, x_{n}(\phi(t))\right)\right)\right) \rightarrow f_{1}\left(t, x\left(g_{1}(t, x(\phi(t)))\right)\right) .
$$

Now by using the continuity of the functions $f_{2}$, assumption (5) and Lebesgues dominated convergence theorem [17], we obtain

$$
\int_{0}^{t} f_{2}\left(t, x_{n}\left(g_{2}\left(t, x_{n}(\phi(t))\right)\right)\right) d s \rightarrow \int_{0}^{t} f_{2}\left(t, x\left(g_{2}(t, x(\phi(t)))\right)\right) d s,
$$


and

$$
\begin{aligned}
\lim _{n \rightarrow \infty}\left(F x_{n}\right)(t) & =\lim _{n \rightarrow \infty} f_{1}\left(t, x_{n}\left(g_{1}\left(t, x_{n}(\phi(t))\right)\right)\right)+\lim _{n \rightarrow \infty} \int_{0}^{t} f_{2}\left(t, x_{n}\left(g_{2}\left(t, x_{n}(\phi(t))\right)\right)\right) d s \\
& =f_{1}\left(t, x\left(g_{1}(t, x(\phi(t)))\right)\right)+\int_{0}^{t} f_{2}\left(t, x\left(g_{2}(t, x(\phi(t)))\right)\right) d s \\
& =(F x)(t) .
\end{aligned}
$$

This proves that the operator $F$ is continuous.

Now all conditions of Schauder fixed point theorem [17] are satisfied, then the operator $F$ has at least one fixed point $x \in S_{L}$. Consequently there exists at leat one solution $x \in C[0, T]$ of Equation (3). This completes the proof.

Now, we introduce the following equivalence theorem.

Theorem 2. Let the assumptions (1)-(8) be satisfied, then the initial value Problem (1)-(2) has at least one positive solution $x \in C[0, T]$.

Proof. Let $x$ be a solution of the Problem (1)-(2). Integrate (1) and substitute by (2), we obtain the integral Equation (3). Let $x$ be a solution of (3) differentiate (3) we obtain (1) and the initial value (2). This proves the equivalence between the Problem (1)-(2) and the integral Equation (3). Then the Problem (1)-(2) has at least one positive solution $x \in C[0, T]$.

\section{Applications}

As application of our results, we introduce the following corollaries;

Corollary 1. Let the assumptions (1) - (8) of Theorem 2 be satisfied, if

(i) $g_{1}(t, x(\phi(t)))=\int_{0}^{\phi(t)} g_{3}(s, x(s)) d s, \quad g_{3}:[0,1] \times[0,1] \rightarrow R^{+}$is continuous and $g_{3}(t, x(t)) \leq 1$,

(ii) $g_{2}(t, x(t))=x(t)$.

Then the initial value problem

$$
\begin{aligned}
& \frac{d}{d t}\left[x(t)-f_{1}\left(t, x\left(\int_{0}^{\phi(t)} g_{3}(s, x(s)) d s\right)\right)\right]=f_{2}(t, x(x(\phi(t)))), \text { a.e. } t \in(0,1], \\
& x(0)=f_{1}(0, x(0))
\end{aligned}
$$

has at least one positive solution $x \in C[0, T]$.

Corollary 2. Let the assumptions of Theorem 1 be satisfied with $g_{1}(t, x(\phi(t)))=\phi(t)$ and $g_{2}(t, x(\phi(t)))=x(\phi(t))$. Then the integral equation

$$
x(t)=f_{1}(t, x(\phi(t)))+\int_{0}^{t} f_{2}(s, x(x(\phi(s)))) d s, \quad t \in[0, T]
$$

has at least one solution $x \in C[0, T]$. Consequently the initial value problem

$$
\frac{d}{d t}\left[x(t)-f_{1}(t, x(\phi(t)))\right]=f_{2}(t, x(x(\phi(t)))) \text {, a.e. } t \in[0, T]
$$

with the initial data $x(0)=f_{1}(0, x(0))$ has at least one positive solution $x \in C[0, T]$.

Corollary 3. Let the assumptions of Corollary 2 be satisfied with $f_{1}(t, x)=t$, then the initial value problem

$$
\begin{aligned}
\frac{d x(t)}{d t} & =f(t,(x(x(\phi(t))))) \text { a.e, } t \in(0, T], \\
x(0) & =0
\end{aligned}
$$


has at least one positive solution $x \in C[0, T]$ where $f(t, x)=f_{2}(t, x)+1$.

\section{Uniqueness of the solution}

In this section, we prove the uniqueness of the solution for the Problem (1)-(2). Therefore, we have to assume the following assumptions

$\left(1^{\prime}\right)\left|f_{2}(t, x)-f_{2}(t, y)\right| \leq k_{5}|x-y|$

(2') $\sup _{t}\left|f_{2}(t, 0)\right| \leq A, t \in[0, T]$,

(3') $\left|g_{2}(t, x)-g_{2}(t, y)\right| \leq k_{6}|x-y|$.

Theorem 3. Let the assumptions (1)-(3), (5)-(8) and (1')-(3') be satisfied, if $k_{2}\left(L k_{4}+1\right)+k_{5} T\left(L k_{6}+1\right)<1$, then the initial value Problem (1)-(2) has a unique positive solution $x \in C[0, T]$.

Proof. Assumption (4) of Theorem 1 can be deduced from assumptions $\left(1^{\prime}\right)$ and $\left(2^{\prime}\right)$ as follows

$$
\begin{aligned}
\left|f_{2}(t, x)\right| & \leq k_{5}|x|+\left|f_{2}(t, 0)\right| \\
& \leq k_{5} x+A
\end{aligned}
$$

then we deduce that all assumptions of Theorem 1 are satisfied and the solution of Equation (3) exists. Now let $x, y$ be two solutions of (3), then

$$
\begin{aligned}
|x(t)-y(t)|= & \mid f_{1}\left(t, x\left(g_{1}(t, x(\phi(t)))\right)\right)+\int_{0}^{t} f_{2}\left(s, x\left(g_{2}(s, x(\phi(s)))\right)\right) d s \\
& -f_{1}\left(t, y\left(g_{1}(t, y(\phi(t)))\right)\right)-\int_{0}^{t} f_{2}\left(s, y\left(g_{2}(s, y(\phi(s)))\right)\right) d s \mid \\
\leq & \left|f_{1}\left(t, x\left(g_{1}(t, x(\phi(t)))\right)\right)-f_{1}\left(t, y\left(g_{1}(t, y(\phi(t)))\right)\right)\right| \\
& +\int_{0}^{t}\left|f_{2}\left(s, x\left(g_{2}(s, x(\phi(s)))\right)\right)-f_{2}\left(s, y\left(g_{2}(s, y(\phi(s)))\right)\right)\right| d s \\
\leq & k_{2}\left|x\left(g_{1}(t, x(\phi(t)))\right)-y\left(g_{1}(t, y(\phi(t)))\right)\right| \\
& +k_{5} \int_{0}^{t}\left|x\left(g_{2}(s, x(\phi(s)))\right)-y\left(g_{2}(s, y(\phi(s)))\right)\right| d s .
\end{aligned}
$$

But

$$
\begin{aligned}
& \left|x\left(g_{1}(t, x(\phi(t)))\right)-y\left(g_{1}(t, y(\phi(t)))\right)\right| \\
& =\left|x\left(g_{1}(t, x(\phi(t)))\right)-x\left(g_{1}(t, y(\phi(t)))\right)+x\left(g_{1}(t, y(\phi(t)))\right)-y\left(g_{i}(t, y(\phi(t)))\right)\right| \\
& \leq\left|x\left(g_{1}(t, x(\phi(t)))\right)-x\left(g_{1}(t, y(\phi(t)))\right)\right|+\left|x\left(g_{1}(t, y(\phi(t)))\right)-y\left(g_{1}(t, y(\phi(t)))\right)\right| \\
& \leq L\left|g_{1}(t, x(\phi(t)))-g_{1}(t, y(\phi(t)))\right|+\left|x\left(g_{1}(t, y(\phi(t)))\right)-y\left(g_{1}(t, y(\phi(t)))\right)\right| \\
& \leq L k_{4}\|x-y\|+\|x-y\| \\
& =\left(L k_{4}+1\right)\|x-y\| .
\end{aligned}
$$

Similarly, we can obtain

$$
\left|x\left(g_{2}(t, x(\phi(t)))\right)-y\left(g_{2}(t, y(\phi(t)))\right)\right| \leq\left(L k_{6}+1\right)\|x-y\| .
$$

Now

$$
|x(t)-y(t)| \leq k_{2}\left(L k_{4}+1\right)\|x-y\|+k_{5} \int_{0}^{t}\left(L k_{6}+1\right)\|x-y\| d s,
$$

and

$$
\|x-y\| \leq\left(k_{2}\left(L k_{4}+1\right)+k_{5} T\left(L k_{6}+1\right)\right)\|x-y\| .
$$


Then we deduce that

$$
\left(1-\left(k_{2}\left(L k_{4}+1\right)+k_{5} T\left(L k_{6}+1\right)\right)\right)\|x-y\| \leq 0
$$

and from the assumptions $\left(k_{2}\left(L k_{4}+1\right)+k_{5} T\left(L k_{6}+1\right)\right)<1$ we can obtain $x=y$ and the solution of (3) is unique. Consequently, the initial value Problem (1)-(2) has a unique positive solution $x \in C[0, T]$.

\section{Continuous dependence}

\subsection{Continuous dependence on the function $g_{1}$}

Here we prove that the solution of the Problem (1)-(2) depends continuously on the function $g_{1}$.

Definition 1. The solution of the Problem (1)-(2) depends continuously on the function $g_{1}$, if $\forall \epsilon>0, \exists \delta(\epsilon)>$ 0 such that

$$
\left\|g_{1}-g_{1}^{*}\right\| \leq \delta \Rightarrow\left\|x-x^{*}\right\| \leq \epsilon
$$

where $x^{*}$ is the unique solution of the equation

$$
\frac{d}{d t}\left[x^{*}(t)-f_{1}\left(t, x^{*}\left(g_{1}^{*}\left(t,\left(x^{*}(\phi(t))\right)\right)\right)\right)\right]=f_{2}\left(t, x^{*}\left(g_{2}\left(t, x^{*}(\phi(t))\right)\right)\right), \quad \text { a.e. } t \in[0, T]
$$

with the initial data

$$
x^{*}(0)=f_{1}\left(0, x^{*}\left(g_{1}^{*}\left(0,\left(x^{*}(0)\right)\right)\right)\right) .
$$

Theorem 4. Let the assumptions of Theorem 3 be satisfied, then the solution of initial value Problem (1)-(2) depends continuously on the function $g_{1}$.

Proof. Let $x$ and $x^{*}$ be the solution of the initial value Problems (1)-(2) and (6)-(7) respectively, then we have

$$
\begin{aligned}
\left|x(t)-x^{*}(t)\right|= & \mid f_{1}\left(t, x\left(g_{1}(t, x(\phi(t)))\right)\right)+\int_{0}^{t} f_{2}\left(s, x\left(g_{2}(s, x(\phi(s)))\right)\right) d s \\
& -f_{1}\left(t, x^{*}\left(g_{1}^{*}\left(t, x^{*}(\phi(t))\right)\right)\right)+\int_{0}^{t} f_{2}\left(s, x^{*}\left(g_{2}\left(s, x^{*}(\phi(s))\right)\right)\right) d s \mid \\
\leq & \left|f_{1}\left(t, x\left(g_{1}(t, x(\phi(t)))\right)\right)-f_{1}\left(t, x^{*}\left(g_{1}^{*}\left(t, x^{*}(\phi(t))\right)\right)\right)\right| \\
& +\int_{0}^{t}\left|f_{2}\left(s, x\left(g_{2}(s, x(\phi(s)))\right)\right)-f_{2}\left(s, x^{*}\left(g_{2}\left(s, x^{*}(\phi(s))\right)\right)\right)\right| d s \\
\leq & \left.k_{2} \mid x\left(g_{1}(t, x(\phi(t)))\right)\right)-x^{*}\left(g_{1}^{*}\left(t, x^{*}(\phi(t))\right)\right) \mid \\
& \left.+k_{5} \int_{0}^{t} \mid x\left(g_{2}(s, x(\phi(s)))\right)\right)-x^{*}\left(g_{2}\left(s, x^{*}(\phi(s))\right)\right) \mid d s \\
\leq & \left.k_{2} \mid x\left(g_{1}(t, x(\phi(t)))\right)\right)-x^{*}\left(g_{1}\left(t, x^{*}(\phi(t))\right)\right) \mid \\
& \left.+k_{2} \mid x^{*}\left(g_{1}\left(t, x^{*}(\phi(t))\right)\right)\right)-x^{*}\left(g_{1}^{*}\left(t, x^{*}(\phi(t))\right)\right) \mid+k_{5} T\left(L k_{6}+1\right)\left\|x-x^{*}\right\| \\
\leq & \left.k_{2}\left(L k_{4}+1\right)\left\|x-x^{*}\right\|+k_{2} L \mid g_{1}\left(t, x^{*}(\phi(t))\right)\right)-g_{1}^{*}\left(t, x^{*}(\phi(t))\right) \mid \\
& +k_{5} T\left(L k_{6}+1\right)\left\|x-x^{*}\right\| \\
\leq & \left(k_{2}\left(L k_{4}+1\right)+k_{5} T\left(L k_{6}+1\right)\right)\left\|x-x^{*}\right\|+k_{2} L \delta .
\end{aligned}
$$

Then

$$
\left\|x-x^{*}\right\| \leq \frac{k_{2} L \delta}{\left(1-\left(k_{2}\left(L k_{4}+1\right)+k_{5} T\left(L k_{6}+1\right)\right)\right.}=\epsilon ，
$$

and by the assumption $\left(k_{2}\left(L k_{4}+1\right)+k_{5} T\left(L k_{6}+1\right)\right)<1$, then the solution of (3) depends continuously on the functions $g_{1}$. Consequently the solution of the Problem (1)-(2) depends continuously on the functions $g_{1}$ which complete the proof. 


\subsection{Continuous dependence on the function $g_{2}$}

Here we prove that the solution of the Problem (1)-(2) depends continuously on the function $g_{2}$.

Definition 2. The solution of the Problem (1)-(2) depends continuously on the function $g_{2}$, if $\forall \epsilon>0, \exists \delta(\epsilon)>$ 0 such that,

$$
\left\|g_{2}-g_{2}^{*}\right\| \leq \delta \Rightarrow\left\|x-x^{*}\right\| \leq \epsilon,
$$

where $x^{*}$ is the unique solution of the equation

$$
\frac{d}{d t}\left[x^{*}(t)-f_{1}\left(t, x^{*}\left(g_{1}\left(t,\left(x^{*}(\phi(t))\right)\right)\right)\right)\right]=f_{2}\left(t, x^{*}\left(g_{2}^{*}\left(t, x^{*}(\phi(t))\right)\right)\right), \quad \text { a.e., } t \in[0, T],
$$

with the initial data

$$
x^{*}(0)=f_{1}\left(0, x^{*}\left(g_{1}\left(0,\left(x^{*}(0)\right)\right)\right)\right) .
$$

Theorem 5. Let the assumptions of Theorem 3 be satisfied, then the solution of initial value Problem (1)-(2) depends continuously on the function $g_{2}$.

Proof. The proof follow similarly as the proof of Theorem 4 .

\section{Examples}

Example 1. Consider the following problem

$$
\frac{d}{d t}\left[x(t)-\frac{1}{18}\left(1+t^{2}\right)-\frac{1}{8} x\left(\frac{\beta t}{1+x^{2}(\beta t)}\right)\right]=\frac{1}{7-t}+\frac{e^{-t}}{16} x\left(\frac{x(\beta t) e^{-x^{2}(\beta t)}}{1+\sin ^{2} x(\beta t)}\right)
$$

with the initial data

$$
x(0)=\frac{4}{63} .
$$

where $t \in(0,1]$ and $\beta \in(0,1]$. Here we have

$\phi(t)=\beta t$,

$g_{1}(t, x(\phi(t)))=\frac{\beta t}{1+x^{2}(\beta t)}$,

$g_{1}(t, x) \leq \beta t$,

$f_{1}\left(t, x\left(g_{1}(t, x(\phi(t)))\right)\right)=\frac{1}{18}\left(1+t^{2}\right)+\frac{1}{8} x\left(\frac{\beta t}{1+x^{2}(\beta t)}\right)$,

$f_{1}(t, x)=\frac{1}{18}\left(1+t^{2}\right)+\frac{1}{8} x$,

$g_{2}(t, x(\phi(t)))=\frac{x(\beta t) e^{-x^{2}(\beta t)}}{1+\sin ^{2} x(\beta t)}$

$g_{2}(t, x) \leq x$,

$f_{2}\left(t, x\left(g_{2}(t, x(\phi(t)))\right)\right)=\frac{1}{7-t}+\frac{e^{-t}}{16} x\left(\frac{x(\beta t) e^{-x^{2}(\beta t)}}{1+\sin ^{2} x(\beta t)}\right)$,

$f_{2}(t, x)=\frac{1}{7-t}+\frac{e^{-t}}{16} x$. Thus we have $k_{1}=\frac{1}{9}, k_{2}=\frac{1}{8}, k_{3}=2, k_{4}=2, k_{5}=\frac{1}{16}, A=\frac{1}{6}, M=\frac{11}{48}, L \simeq 0.557<$ 1 and $L T+x(0) \approx 0.62<T=1$.

Now all the assumptions of Theorem 1 are satisfied, then the Problem (10)-(11) has at least one solution $x \in C[0, T]$.

Example 2. Consider the following problem

$$
\frac{d}{d t}\left[x(t)-\frac{1}{48}(1+t)-\frac{1}{4} t^{2} x\left(\frac{\frac{1}{4} t^{2}}{1+32 x\left(t^{2}\right)}\right)\right]=\frac{1}{5+2 t} \sin ^{2}(3(t+1))+\frac{1}{12} x\left(\frac{x\left(t^{2}\right) \sin ^{2}\left(x\left(t^{2}\right)\right)}{1+x^{2}\left(t^{2}\right)}\right),
$$

with the initial data

$$
x(0)=\frac{1}{48},
$$


where $t \in\left(0, \frac{1}{2}\right]$. Here we have

$\phi(t)=t^{2}$,

$g_{1}(t, x(\phi(t)))=\frac{\frac{1}{4} t^{2}}{1+32 x\left(t^{2}\right)}$

$g_{1}(t, x) \leq t^{2}$,

$f_{1}\left(t, x\left(g_{1}(t, x(\phi(t)))\right)\right)=\frac{1}{48}(1+t)+\frac{1}{4} t^{2} x\left(\frac{\frac{1}{4} t^{2}}{1+32 x\left(t^{2}\right)}\right)$,

$f_{1}(t, x)=\frac{1}{48}(1+t)+\frac{1}{4} t^{2} x$,

$g_{2}(t, x(\phi(t)))=\frac{x\left(t^{2}\right) \sin ^{2}\left(x\left(t^{2}\right)\right)}{1+x^{2}\left(t^{2}\right)}$

$g_{2}(t, x) \leq x$,

$f_{2}\left(t, x\left(g_{2}(t, x(\phi(t)))\right)\right)=\frac{1}{5+2 t} \sin ^{2}(3(t+1))+\frac{1}{12} x\left(\frac{x\left(t^{2}\right) \sin ^{2}\left(x\left(t^{2}\right)\right)}{1+x^{2}\left(t^{2}\right)}\right)$,

$f_{2}(t, x)=\frac{1}{5+2 t} \sin ^{2}(3(t+1))+\frac{1}{12} x$. Thus, we have $k_{1}=\frac{7}{48}, k_{2}=\frac{1}{16}, k_{3}=\frac{17}{4}, k_{4}=2, k_{5}=\frac{1}{12}, A=\frac{1}{5}, M=$ $\frac{29}{120}, L \simeq 0.586<1$ and $L T+x(0) \approx 0.3139<T=\frac{1}{2}$.

Now all the assumptions of Theorem 1 are satisfied, then the Problem (12)-(13) has at least one solution $x \in C[0, T]$.

\section{Conclusion}

Here we relaxed the assumptions and generalized the results in $[8,11,14,18]$ and [1]. We proved the existence of at lease one solution of the Problem (1)-(2). The sufficient condition for the uniqueness of the solution have been given and the continuous dependence of the unique solution have been proved. Also some examples and applications have been given.

Author Contributions: All authors contributed equally to the writing of this paper. All authors read and approved the final manuscript.

Conflicts of Interest: "The authors declare no conflict of interest."

\section{References}

[1] Miranda, M., \& Pascali, E. (2006). On a type of evolution of self-referred and hereditary phenomena. Aequationes Mathematicae, 71(3), 253-268.

[2] Tuan, N. M., \& Nguyen, L. T. (2010). On solutions of a system of hereditary and self-referred partial-differential equations. Numerical Algorithms, 55(1), 101-113.

[3] Van Le, U., \& Nguyen, L. T. (2008). Existence of solutions for systems of self-referred and hereditary differential equations. Electronic Journal of Differential Equations, 51, 1-7.

[4] Yang, D., \& Zhang, W. (2004). Solutions of equivariance for iterative differential equations. Applied Mathematics Letters, 17(7), 759-765.

[5] Anh, P. K., Lan, N. T. T., \& Tuan, N. M. (2012). Solutions to systems of partial differential equations with weighted self-reference and heredity. Electronic Journal of Differential Equations, 2012(117), 1-14.

[6] Berinde, V. (2010). Existence and approximation of solutions of some first order iterative differential equations. Miskolc Mathematical Notes, 11(1), 13-26.

[7] Buica, A. (1995). Existence and continuous dependence of solutions of some functional-differential equations. In Babes-Bolyai Univ., Cluj-Napoca, Seminar on fixed point theory, 3, 1-13.

[8] Eder, E. (1984). The functional differential equation $x^{\prime}(t)=x(x(t))$. Journal of Differential Equations, 54(3), 390-400.

[9] El-Sayed, A. M. A., \& Ahmed, R. G. (2020). Solvability of the functional integro-differential equation with self-reference and state-dependence. Journal of Nonlinear Sciences \& Applications, 13(1), 1-8.

[10] El-Sayeda, A. M. A., El-Owaidyb, H., \& Ahmedb, R. G. (2020). Solvability of a boundary value problem of self-reference functional differential equation with infinite point and inte-gral conditions. Journal of Mathematics and Computer Science, 21, 296-308.

[11] EL-Sayed, A. M. A., \& Ebead, H. R. (2020). On an initial value problem of delay-refereed differential Equation. International Journal of Mathematics Trends and Technology, 5(66), 32-37.

[12] El-Sayed, A., Hamdallah, E. M. \& Ebead, H. R. (2020). Positive solutions of an initial value problem of a delay-self-reference nonlinear differential equation. Malaya Journal of Matematik, 8(3), 1001-1006.

[13] El-Sayed, A., \& Ebead, H. R. (2020). On the solvability of a self-reference functional and quadratic functional integral equations. Filomat, 34(1), 129-141. 
[14] Fečkan, M. (1993). On a certain type of functional differential equations. Mathematica Slovaca, 43(1), 39-43.

[15] Gal, C. G. (2007). Nonlinear abstract differential equations with deviated argument. Journal of Mathematical Analysis and Applications, 333(2), 971-983.

[16] Letelier, J. C., Kuboyama, T., Yasuda, H., Cárdenas, M. L., \& Cornish-Bowden, A. (2005). A self-referential equation, $f(f)=f$, obtained by using the theory of $(m ; r)$ systems: Overview and applications. Algebraic Biology, 115-126.

[17] Goebel, K., \& Kirk, W. A. (1990). Topics in metric fixed point theory (Vol. 28). Cambridge University Press.

[18] Lan, N. T. \& Pascali, E. (2018). A two-point boundary value problem for a differential equation with self-refrence, Electron Journal of Mathematical Analysis and Applications, 6(1), 25-30.

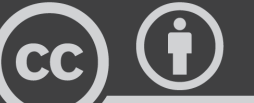

BY

(C) 2020 by the authors; licensee PSRP, Lahore, Pakistan. This article is an open access article distributed under the terms and conditions of the Creative Commons Attribution (CC-BY) license (http://creativecommons.org/licenses/by/4.0/). 